\title{
OURICURI
}

\section{HERPETOFAUNA DE UMA ÁREA SERRANA, SANTA CRUZ DA BAIXA VERDE, PERNAMBUCO}

\author{
Ana Maria Siqueira QUIRINO'; Gleymerson Vieira Lima de ALMEIDA²; Ednilza Maranhão dos \\ SANTOS3; Mauro de Melo JÚNIOR ${ }^{4}$
}

\begin{abstract}
${ }^{1}$ Bolsista CNPq, Universidade Federal Rural de Pernambuco/Unidade Acadêmica de Serra Talhada. Avenida Gregório Ferraz Nogueira, S/N, Bairro: José Tomé de Souza Ramos, Serra Talhada, Pernambuco, Brasil. CEP: 56909-535. E-mail: anasiqueira17@hotmail.com.

2Professor na Faculdade de Integração do Sertão. Rua João Luiz de Melo, 2110, Bairro Tancredo Neves, Serra Talhada, Pernambuco, Brasil.

${ }^{3}$ Professor Adjunto do Departamento de Biologia, Universidade Federal Rural de Pernambuco. Recife, Pernambuco, Brasil. Autor para correspondência: ednilzamaranhao@gmail.com.

${ }^{4}$ Professor Adjunto do Departamento de Biologia, da Universidade Federal Rural de Pernambuco (DB/UFRPE).

http://doi.org/10.29327/ouricuri.v8.i1.a1
\end{abstract}

\begin{abstract}
Resumo: Este trabalho objetivou inventariar as espécies de anfíbios e répteis no sítio Olho d'Água, Santa Cruz da Baixa Verde, Pernambuco, destacando sua ocupação nos diferentes hábitats e microhabitats, bem como seu status de conservação. A coleta dos dados foi realizada em agosto de 2010 a junho de 2011, através de buscas ativas e passivas, utilizando armadilhas de interceptação e queda - modelo padrão pit fall traps, nos períodos diurnos e noturnos nos sítios refúgio, alimentação e procriação desses táxons. Foram registradas 19 espécies de anfíbios distribuídas em seis famílias, todas pertencentes à ordem Anura: Bufonidae (2), Hylidae (5), Leiuperidae (1), Leptodactylidae (8), Pipidae (1) e Cycloramphidae (2) e 15 espécies de répteis distribuídos entre 10 famílias, essas pertencentes à Tropiduridae (2), Gekkonidae (1), Teiidae (2), Iguanidae (1), Gymnophthalmidae (1), Leiosauridae (1), Sphaerodactylidae (1), Phylloctylidae (1), Dipsadidae (4) e Viperidae (1). A lista apresentada no presente trabalho é bastante representativa do ponto de vista regional e principalmente no que se refere ao semiárido brasileiro, destacando elementos de áreas mésicas e xéricas. A comunidade da herpetofauna para a localidade estudada é composta por espécies típicas de Caatinga com alguns elementos de área úmida.
\end{abstract}

Palavras-chaves: Anfíbios; Répteis; Conservação da Caatinga.

\section{HERPETOFAUNA OF A MOUNTAIN AREA, SANTA CRUZ DA BAIXA VERDE, PERNAMBUCO}

Abstract: This work aimed to inventory the species of amphibians and reptiles in the Olho d'Água site, Santa Cruz of Baixa Verde/Pernambuco, highlighting its occupation in the different habitat and microhabitat, as well as its conservation status. The collection of data was carried out in august 2010 until june 2011 through active and passive searches using interception traps and standard fall-model pit fall traps, daytime and nocturnal periods in refuge, feeding and breeding sites of these taxa. It was recorded 19 species of amphibians distributed in six families, all belonging to the order Anura: Bufonidae (2), Hylidae (5), Leiuperidae (1), Leptodactylidae (8), Pipidae (1) and Cycloramphidae (2) and 15 species of reptiles distributed between 10 families, these belonging to the Tropiduridae (2), Geckos (1), Teiidae (2), Iguanidae (1), Gymnophthalmidae (1), Leiosauridae (1), Sphaerodactylidae (1), Phylloctylidae (1), Dipsadidae (4) and Viperidae (1). The list presented in the present work is fairly representative from the regional point of view and mainly as regards 
the Brazilian semi-arid, highlighting elements of mésicas and xéricas areas. The community of herpetofauna for the studied locale is composed of typical Caatinga species with some elements of moist area.

Keywords: Amphibian; Reptiles; Caatinga of Conservation.

\section{INTRODUÇÃO}

O semiárido nordestino apresenta diferentes paisagens, dentre essas o maior destaque é para várias feições de Caatingas, apresentando áreas xéricas e algumas mésicas. De uma maneira geral a Caatinga, bioma exclusivamente brasileiro, apresenta uma ampla diversidade biológica, porém é o mais ameaçado, menos estudado e o menos protegido em unidades de conservação (RODRIGUES, 2003a, 2003b).

Um dos principais problemas enfrentados na região semiárida tem sido a degradação e destruição dos recursos naturais, causados principalmente pela ação antrópica. Isso acarreta em problemas irreparáveis nas diferentes paisagens distintas desse Bioma, principalmente quando se refere à fauna silvestre. Estudos para a Caatinga revelam uma biodiversidade bastante expressiva, todavia essa informação é bastante pontual o que impossibilita ações de conservação mais direcionadas e consequentemente a criação de políticas ambientais locais e estaduais.

Os brejos de altitudes são áreas que apresentam características peculiares, tais como altitudes maiores que $600 \mathrm{~m}$, altas taxas de precipitação (900 - $12000 \mathrm{~mm}$ ), clima úmido ou semiúmido, solos profundos, vegetação compostas por floresta perenifólia ou subperenifólia, xerófila de caatinga, florestas decíduas (PÔRTO et al., 2004; ANDRADE-LIMA, 1960), o que a tornam uma área de exceção úmida e isolada no semiárido nordestino, evidenciando sua importância como áreas de preservação permanente.

Rodrigues (2003a, 2003b) relatou a existência de 52 espécies de anfíbios e 150 de répteis para as áreas do semiárido Nordestino, com ênfase as áreas de Caatingas. Em Pernambuco, Moura (2011) através de uma compilação de diferentes estudos realizados no estado, lista para o Estado cerca de 187 espécies, dos quais 71 (37,63\%) são anfíbios e 116 (62,37\%) répteis para um total de 43 localidades, porém nenhuma informação para o município de Santa Cruz da Baixa Verde, Sertão do Pajeú, Pernambuco.

O objetivo desse trabalho foi inventariar espécies de anfíbios e répteis, destacando sua ocupação nos diferentes hábitats e microhábitats, frequência de ocorrência e status de conservação em uma área serrana no Município de Santa Cruz da Baixa Verde, Pernambuco.

\section{MATERIAL E MÉTODOS \\ ÁREA DE ESTUDO}

O Sítio Olho d'Água situa-se no município de Santa Cruz da Baixa Verde - PE posicionada a uma latitude de $07^{\circ} 49^{\prime} 19^{\prime \prime}$ Oeste e $38^{\circ} 09^{\prime} 10^{\prime \prime}$ ao Sul. Está localizada na mesorregião do 
Sertão de Pernambuco e na microrregião do Pajeú, limitando-se ao Norte com o estado da Paraíba, ao Sul com o município de Calumbí e a Oeste com a cidade de Serra Talhada (Figura 1).

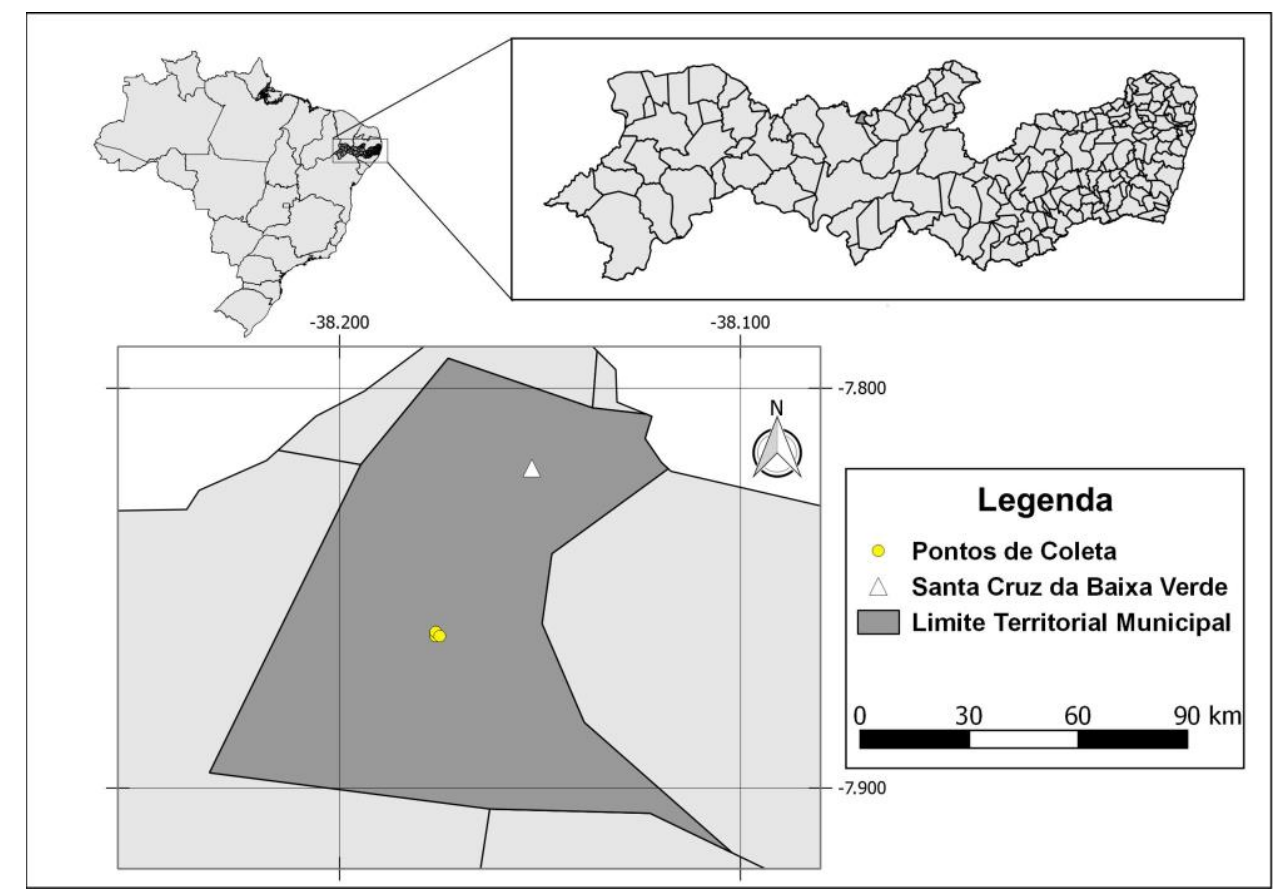

Figura 1. Localização do Sítio Olho d'Água, Santa Cruz da Baixa - Pernambuco, com destaque para os pontos de coletas. Fonte: ALMEIDA, 2017.

O local possui 850 metros de elevação, estando localizada cerca de $445 \mathrm{~km}$ da capital do Estado, Recife. Possui temperatura média anual de $21,9^{\circ} \mathrm{C}$, clima tropical chuvoso e uma área de 90,8 km² (INSTITUTO BRASILEIRO DE GEOGRAFIA E ESTATÍSTICA, 2015).

A localidade referida está inserida em um vale com encostas bastante acentuada, incluindo paredões rochosos e pequenas quedas d'água que the confere um clima mais úmido na paisagem local. A vegetação é bastante variada com elementos de caatinga arbustiva e em alguns pontos, nas serras, encontra-se uma vegetação mais arbórea (PÔRTO et al., 2004). A presença de diferentes corpos d'água (riacho, cachoeira e tanques) destaca a relevância dessa área, sendo estes locais propícios para os anfíbios e algumas espécies de répteis (RODRIGUES, 2003b; FREITAS e SILVA, 2007).

No presente estudo foram selecionados cinco pontos fixos (interior de mata), amostrados através de armadilhas de interceptação e queda - método passivo de coleta, e três áreas abertas para buscas ativas (Tabela 1).

As coletas dos dados foram realizadas mensalmente de agosto de 2010 a junho de 2011, durante seis dias através de buscas passivas utilizando armadilhas de interceptação e queda modelo padrão pit fall traps (NOGUEIRA et al., 2005) em forma de "Y" adaptado para captura de anfíbios e répteis, e buscas ativas, por meio de caminhadas esporádicas nos corpos d'água (Figura 2). 
Tabela 1. Pontos de coletas no Sítio Olho d'Água, Santa Cruz da Baixa/Pernambuco, em agosto de 2010 a junho de 2011.

\begin{tabular}{|c|c|c|c|}
\hline Área & Coordenada & Ambientes & Descrição geral \\
\hline $\begin{array}{l}\text { Caatinga } \\
\text { aberta }\end{array}$ & $\begin{array}{l}07^{\circ} 51^{\prime} 44,9 " \mathrm{~S} \\
38^{\circ} 10^{\prime} 33.7^{\prime \prime} \mathrm{W} \\
07^{\circ} 51^{\prime} 42.3 \text { " S } \\
38^{\circ} 10^{\prime} 35.4^{\prime \prime} \mathrm{W} \\
07^{\circ} 51^{\prime} 45.2 \text { ' S } \\
38^{\circ} 10^{\prime} 34.8^{\prime \prime} \mathrm{W}\end{array}$ & $\begin{array}{l}\text { Cacimbão, } \\
\text { Tanque I e II }\end{array}$ & $\begin{array}{c}\text { Vegetação herbácea, com elementos } \\
\text { arbustivos e arbóreos, em ambiente de } \\
\text { pastagem, com enclaves de floresta em um } \\
\text { dos lados da área. }\end{array}$ \\
\hline $\begin{array}{l}\text { Enclaves de } \\
\text { mata }\end{array}$ & $\begin{array}{l}07^{\circ} 51^{\prime} 40.2 " \mathrm{~S} \\
38^{\circ} 10^{\prime} 35.4^{\prime \prime} \mathrm{W} \\
07^{\circ} 51^{\prime} 42.1^{\prime \prime} \mathrm{S} \\
38^{\circ} 10^{\prime} 36.2^{\prime \prime} \mathrm{W}\end{array}$ & $\begin{array}{l}\text { Riacho e } \\
\text { bordas do } \\
\text { riacho }\end{array}$ & $\begin{array}{l}\text { Vegetação herbácea, bromeliáceas, arbustiva/ } \\
\text { arbórea, em ambiente de pastagem, com } \\
\text { elementos arbóreos típicos de brejos. }\end{array}$ \\
\hline Antropizada & $\begin{array}{l}07^{\circ} 51^{\prime} 45.0 " \mathrm{~S} \\
38^{\circ} 10^{\prime} 39.0^{\prime \prime} \mathrm{W}\end{array}$ & Área irrigada & $\begin{array}{l}\text { Vegetação arbustiva, com predominância de } \\
\text { frutíferas. }\end{array}$ \\
\hline
\end{tabular}
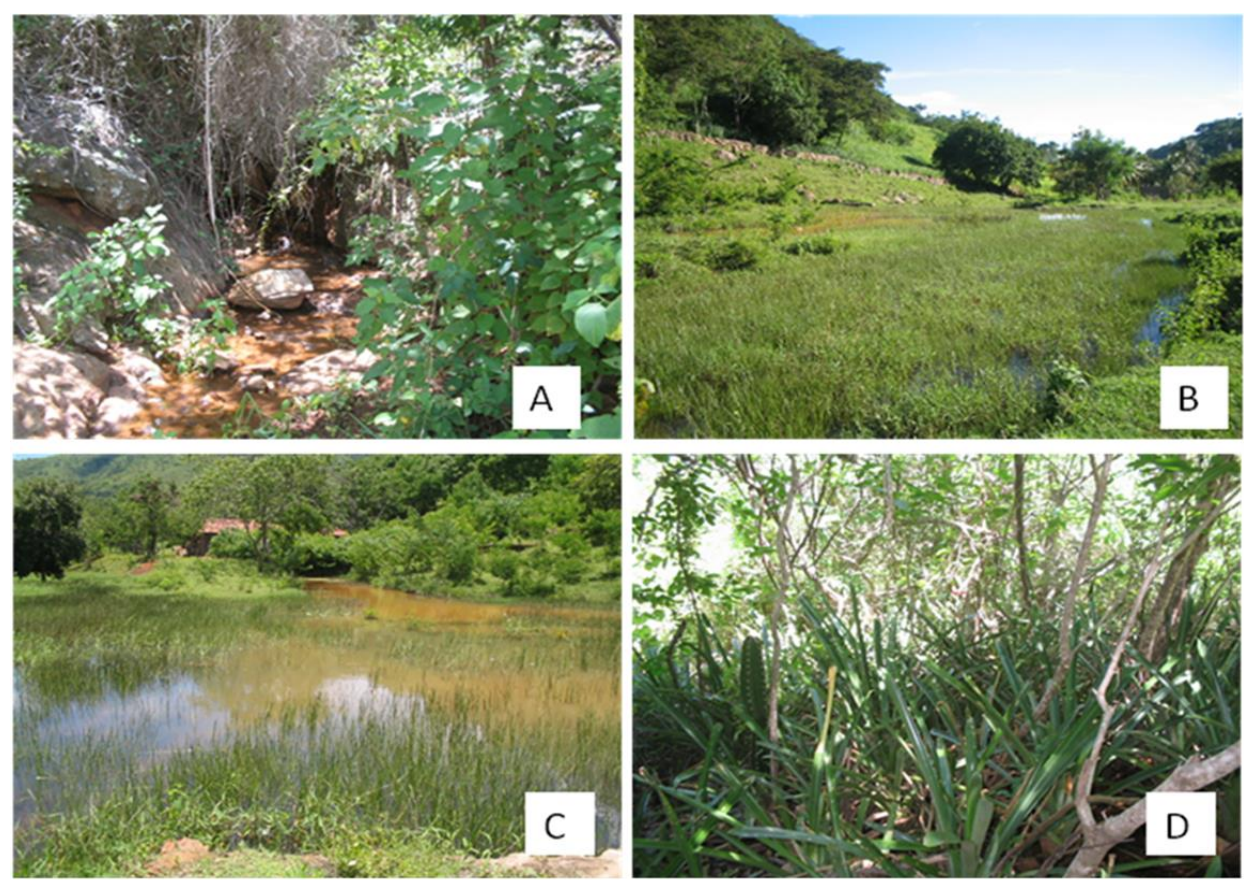

Figura 2. Corpos de água que compõem a paisagem do Sítio Olho d’Água, Santa Cruz da Baixa - Pernambuco. A- Riacho; B- Área alagada próxima à encosta; C- Lago; D- vegetação do fragmento, destacando bromeliáceas. Fonte: SANTOS, 2010.

\section{Coleta dos dados}

Para material testemunho, pelo menos dois exemplares de cada espécie foram sacrificados utilizando-se anestésico (@Ketalar), supervisionado por um médico veterinário, sob 
autorização do IBAMA (Reg. № 11218-1), encontra-se depositado na Coleção Herpetológica da Universidade Federal Rural de Pernambuco.

\section{Análises dos dados}

Para a análise dos dados utilizou-se estatística descritiva como frequência dos registros e para a constância de ocorrência de cada espécie utilizou-se o método de Dajoz (1983), que tem a seguinte expressão matemática: $C=$ p.100/P. Onde: $C=$ constância de ocorrência de cada espécie; $\mathrm{P}=$ número de excursões em que a espécie foi registrada; $\mathrm{P}=$ número total de excursões. A análise define as seguintes categorias de espécies: constantes (ocorreram em mais de $50 \%$ das amostras), acessórias (ocorreram entre 25 e $50 \%$ das amostras) e acidentais (ocorreram em menos de $25 \%$ da amostra).

\section{RESULTADOS E DISCUSSÕES}

O esforço de coleta foi diluído em 162 horas/homem de busca ativa e 432 horas de busca passiva, com dois observadores em cada coleta, o que resultou um registro de 19 espécies de anfíbios distribuídas em seis famílias, todas pertencentes à ordem Anura: Bufonidae (2), Hylidae (5), Leiuperidae (1), Leptodactylidae (8), Pipidae (1) e cycloramphidae (2) e 15 espécies de répteis distribuídos entre 10 famílias, essas pertencentes à Tropiduridae (2), Gekkonidae (1), Teiidae (2), Iguanidae (1), Gymnophthalmidae (1), Leiosauridae (1), Sphaerodactylidae (1), Phylloctylidae (1), Dipsadidae (4) e Viperidae (1) (Tabela 2).

Todas as espécies listadas para localidade já foram registradas em outros trabalhos realizados em áreas de Caatinga, para Pernambuco (SANTOS e CARNAVAL, 2001; RODRIGUES, 2003a, 2003b).

As famílias Hylidae e Leptodactylidae tiveram uma maior representatividade com cinco e oito espécies respectivamente. Do total de espécies de anfíbios registradas, 18 ocorreram na área de caatinga aberta (1) e apenas Odontophrynus carvalhoi e Proceratophrys cristiceps foram observadas na área de enclaves de mata (2). Quanto aos répteis à família Tropiduridae com registro de duas espécies Tropidurus hispidus (Spix, 1825) e Tropidurus semitaeniatus (Spix, 1825), obtiveram uma maior representatividade com $22 \%$ dos indivíduos contabilizados, seguidos de Gekkonidae (11\%), Teiidae (11\%), Sphaerodactylidae (11\%), Iguanidae (11\%) e Gymnophthalmidae (11\%), Leiosauridae (11\%) e Phyllodactylidae (11\%). Em relação as espécies de serpentes registradas, foram observadas duas famílias: Dipsadidae (4) e Viperidae (1), onde a família Dipsadidae (75\%) obteve maior representatividade entre as serpentes e Viperidae representando apenas $25 \%$ da amostra.

O número de espécies amostrado é similar aos trabalhos realizados no semiárido (ARZABE, 1999; RODRIGUES 2003a, 2003b, 2005; ARZABE et al., 2005; BORGES-NOJOSA e SANTOS, 2005; MOURA, 2011) especialmente para a Caatinga (RODRIGUES 2003a, 2003b, 2005; VIEIRA et al., 2007; LOEBMANN e HADDAD, 2010; MOURA et al., 2011). No entanto, os 
trabalhos realizados em brejos apresentam resultados diferentes, pois, se tem uma maior riqueza de anfíbios (cerca de 10 a 37 espécies de anuros) e menor riqueza de répteis, com 12 espécies (MOURA et al., 2011).

O destaque dessa maior riqueza dos brejos é o registro da anurofauna do Complexo do Planalto da lbiapaba (CE), com 37 espécies (LOEBMANN e HADDAD, 2010). Segundo esses autores, esse Complexo é considerado o local com a maior diversidade entre os brejos nordestinos. Essa diversidade pode estar relacionada a fatores ecológicos, históricos, bem como por se tratar de uma área ainda bem preservada do ponto de vista ambiental.

Foram estudadas áreas de Caatinga aberta, enclaves de mata e uma área antropizada, sendo que dentro da área de Caatinga aberta os ambientes hídricos que compunha o ambiente foram um cacimbão, tanque I e II. Na área de enclaves de mata continha um riacho intermitente e suas bordas também foram pesquisadas. Já na área antropizada, o ambiente hídrico analisado foi à área irrigada.

Das áreas estudadas, quem apresentou os maiores números de espécies foram os enclaves de mata $(n=28)$, seguida pela área Caatinga aberta $(n=9)$ e a área antropizada $(n=6)$.

A ocupação espacial demonstrou certo grau de relação com as fitofisionomias das áreas amostradas, uma vez que a maioria das espécies foram encontradas na área de Caatinga aberta, preferindo vegetação arbustiva o que pode está relacionado a maior variedade de microhabitats disponíveis neste ambiente.

Como áreas de Caatinga aberta geralmente apresentam uma diversidade de recursos, com ambientes aquáticos com diferentes extratos vegetacionais, esses táxons possivelmente encontraram condições mais favoráveis nessa área para ocupação. Quanto à frequência de ocorrência, foram consideradas acidentais um total de duas espécies, sete foram acessórias e 10 constantes (Tabela 2).

Quanto aos anfíbios, a família Hylidae apresentou uma maior riqueza e também uma maior abundância, onde as espécies com maior frequência de ocorrência foram Phyllomedusa nordestina, Scinax x-signatus, Rhinella jimi e Hypsiboas raniceps.

Com base na União Internacional para a Conservação da Natureza (IUCN, 2015), no Instituto Chico Mendes de Conservação da Biodiversidade (ICMBIO, 2014) e na Secretaria de Meio Ambiente e Sustentabilidade de Pernambuco (SEMAS, 2015) dessas espécies registradas nenhuma delas encontra-se na lista de espécies ameaçadas de extinção, todavia a maioria encontra-se como status de conservação desconhecido (IUCN, 2015).

Loebmann e Haddad (2010) destacaram nas suas análises Odontophrynus carvalhoi como uma espécie mais vulnerável a alterações no seu habitat resultantes da ação antrópica. O registro deste táxon em Santa Cruz da Baixa Verde é classificado como constante (mais de 50\%) nas coletas realizadas em buscas ativas e passivas. 
Tabela 2. Abundância e ocorrência das espécies de anfíbios e répteis amostrados no Sítio Olho d'Água, Santa Cruz da Baixa Verde/PE no período de agosto/2010 a junho /2011. Área: A- área antropizada, B- enclaves de mata, C- Caatinga aberta. Ambientes hídricos: 1- área irrigada, 2riacho, 3- bordas do riacho, 4- cacimbão, 5- tanque I, 6- taque II. Frequência de ocorrência (FO): Constante - mais de 50\%, acessória - entre 25 e 50\% e acidental - menos de $25 \%$. Forma de Registro: BA - busca ativa, BP - busca passiva.

\begin{tabular}{|c|c|c|c|}
\hline Subclasse/ Ordem/ Família/ Espécie & Habitat/Microhabit & $\mathrm{FO}$ & $\begin{array}{l}\text { Forma de } \\
\text { Registro }\end{array}$ \\
\hline \multirow{2}{*}{$\begin{array}{l}\text { LISSAMPHIBIA/ANURA/BUFONIDAE } \\
\text { Rhinella granulosa Spix, 1824 } \\
\text { Rhinella jimi (Stevaux, 2002) }\end{array}$} & $A, 1$ & Acessória & BA \\
\hline & $A, 1$ & Constante & BA \\
\hline \multirow{2}{*}{$\begin{array}{l}\text { CYCLORAMPHIDAE } \\
\text { Odontophrynus carvalhoi Savage \& Cei, } 1965 \\
\text { Proceratophrys cristiceps (Müller, } 1884 \text { "1883") }\end{array}$} & $\mathrm{B}, 3$ & Constante & $\mathrm{BP}$ \\
\hline & $\mathrm{B}, 3$ & Acessória & $\mathrm{BP}$ \\
\hline \multirow{3}{*}{$\begin{array}{l}\text { HYLIDAE } \\
\text { Dendropsophus gr. nanus (Boulenger, 1889) } \\
\text { Dendropsophus oliveirai Bokermann, } 1963 \\
\text { Dendropsophus sp. } \\
\text { Hypsiboas crepitans Wied-Neuwied, } 1824 \\
\text { Hypsiboas raniceps (Cope, 1862) } \\
\text { Phyllomedusa nordestina (Caramaschi 2006) } \\
\text { Scinax x-signatus (Spix, 1824) }\end{array}$} & $\mathrm{B}, 2,3$ & Constante & BA \\
\hline & $\begin{array}{c}B, 2,3 \\
B, 2,3 \\
C, 1 \\
C, 1 \\
C, 1,5,6\end{array}$ & $\begin{array}{l}\text { Constante } \\
\text { Acidental } \\
\text { Acessória } \\
\text { Constante } \\
\text { Constante }\end{array}$ & $\begin{array}{l}\text { BA } \\
\text { BA } \\
\text { BA } \\
\text { BA } \\
\text { BA }\end{array}$ \\
\hline & $C, 1,5,6$ & Constante & BA \\
\hline \multirow{2}{*}{$\begin{array}{l}\text { LEIUPERIDAE } \\
\text { Physalaemus cuvieri Fitzinger, 1826 } \\
\text { Physalaemus albifrons (Spix, 1824) }\end{array}$} & $\mathrm{B}, 3$ & Constante & $\mathrm{BP}, \mathrm{BA}$ \\
\hline & $\mathrm{B}, \mathrm{C}, 1,3$ & Acessória & BA \\
\hline \multirow{3}{*}{$\begin{array}{l}\text { LEPTODACTYLIDAE } \\
\text { Leptodactylus troglodytes Lutz, } 1830 \\
\text { Leptodactylus vastus Spix, } 1824 \\
\text { Leptodactylus caatingae Heyer \& Juncá, } 2003 \\
\text { Leptodactylus latrans (Steffen, 1815) } \\
\text { Leptodactylus fuscus (Schneider, 1799) }\end{array}$} & $B, 2,3$ & Constante & BA \\
\hline & $\begin{array}{l}\mathrm{B}, 2,3 \\
\mathrm{~B}, 3 \\
\mathrm{~B}, 3\end{array}$ & $\begin{array}{l}\text { Constante } \\
\text { Acidental } \\
\text { Acidental }\end{array}$ & $\begin{array}{l}\text { BA } \\
\text { BA }\end{array}$ \\
\hline & $\mathrm{B}, 2,3$ & Acessória & BA \\
\hline $\begin{array}{l}\text { PIPIDAE } \\
\text { Pipa carvalhoi (Miranda-Ribeiro, 1937) }\end{array}$ & $\mathrm{C}, 4$ & Acessória & BA \\
\hline $\begin{array}{l}\text { SQUAMATA/LIZARDS/IGUANIDAE } \\
\text { Iguana iguana (Linnaeus, 1758) }\end{array}$ & $\mathrm{B}, 3$ & Acidental & BA \\
\hline $\begin{array}{l}\text { LEIOSAURIDAE } \\
\text { Enyalius bibronii Boulenger, } 1885\end{array}$ & $\mathrm{~B}, 3$ & Acidental & $\mathrm{BA}, \mathrm{BP}$ \\
\hline \multirow{2}{*}{$\begin{array}{l}\text { TROPIDURIDAE } \\
\text { Tropidurus hispidus (Spix, 1825) } \\
\text { Tropidurus semitaeniatus (Spix, 1825) }\end{array}$} & $A, B, C, 1,3,4$ & Acessória & BA \\
\hline & $\mathrm{A}, \mathrm{B}, \mathrm{C}, 1,3,4$ & Acessória & BA \\
\hline $\begin{array}{l}\text { GEKKONIDAE } \\
\text { Hemidactylus brasilianus (Amaral, 1935) }\end{array}$ & $A, B, C, 1,3,4$ & Acessória & BA \\
\hline \multirow{2}{*}{$\begin{array}{l}\text { PHYLLODACTYLIDAE } \\
\text { Gymnodactylus geckoides Spix, } 1825 \\
\text { Phyllopezus pollicaris Spix, } 1825\end{array}$} & $\mathrm{~B}, 3$ & Acidental & BA \\
\hline & $\mathrm{B}, 3$ & Acidental & BA \\
\hline $\begin{array}{l}\text { SPHAERODACTYLIDAE } \\
\text { Coleodactylus meridionalis (Boulenger, 1888) }\end{array}$ & $\mathrm{B}, 3$ & Acidental & BA \\
\hline
\end{tabular}


Tabela 2. Continuação

\begin{tabular}{|c|c|c|c|}
\hline Subclasse/ Ordem/ Família/ Espécie & Habitat/Microhabit & FO & $\begin{array}{l}\text { Forma de } \\
\text { Registro }\end{array}$ \\
\hline $\begin{array}{l}\text { TEIIDAE } \\
\text { Ameiva ameiva (Linnaeus, 1758) }\end{array}$ & $\mathrm{B}, 3$ & Acidental & $\mathrm{BP}$ \\
\hline Cnemidophorus cf. ocellifer (Spix, 1825) & $B, 3$ & Acidental & BA \\
\hline $\begin{array}{l}\text { GYMNOPHTHALMIDAE } \\
\text { Anotosaura vanzolinia Dixon, } 1974\end{array}$ & $\mathrm{~B}, 3$ & Acidental & BP \\
\hline $\begin{array}{l}\text { SQUAMATA/SNAKES/ DIPSADIDAE } \\
\text { Oxyrhopus trigeminus Duméril, Bibron \& } \\
\text { Duméril, } 1854\end{array}$ & $\mathrm{~B}, 3$ & Acidental & BA \\
\hline Thamnodynastes hypoconia (Cope, 1860) & $B, 3$ & Acidental & BA \\
\hline $\begin{array}{l}\text { Thamnodynastes sertanejo Bailey, Thomas \& } \\
\text { Silva-Jr, } 2005\end{array}$ & $\mathrm{~B}, 3$ & Acidental & BA \\
\hline Thamnodynastes sp. & $\mathrm{B}, 3$ & Acidental & BA \\
\hline $\begin{array}{l}\text { VIPERIDAE } \\
\text { Caudisona durissa (Linnaeus, 1758) }\end{array}$ & $\mathrm{B}, 3$ & Acidental & BA \\
\hline
\end{tabular}

A lista apresentada no presente trabalho é bastante representativa do ponto de vista regional e principalmente no que se refere ao semiárido brasileiro, destacando elementos de áreas mésicas e xéricas.

Através desse diagnóstico podemos inferir que a busca ativa foi mais eficiente no registro da herpetofauna, todavia foi importante a utilização de um segundo método (armadilhas de interceptação e queda) para maximizar os resultados, tendo em vista que existem espécies que habitam a serrapilheira e são difíceis de serem documentadas nas buscas ativas.

Atualmente é necessário, dentro do bioma Caatinga, uma maior atenção as áreas de encosta como áreas de preservação permanente. Com tudo isso esse trabalho deve contribuir e ajudar os gestores municipais a terem maior atenção às áreas serranas.

\section{CONCLUSÃO}

A área de Caatinga arbustiva-arbórea apresentou uma maior riqueza e uma maior abundância de anfíbios anuros, possivelmente por apresentar uma maior quantidade de corpos d'água.

A fauna de répteis do Sítio Olho d' Água é caracterizada por predomínio de animais da Caatinga e pelo registro de uma espécie de lagarto do domínio atlântico (Coleodactilus meridiolalis).

A área de Caatinga onde se encontra lajedos e rochas em um terreno acidentado apresentou uma maior riqueza de animais.

A comunidade da herpetofauna para a localidade estudada é composta por espécies típicas de Caatinga com alguns elementos de área úmida. 


\section{AGRADECIMENTOS}

Ao Conselho Nacional de Desenvolvimento Científico e Tecnológico (CNPq) pela concessão da bolsa a primeira autora. Aos proprietários das localidades pesquisadas, Manoel Silva, Maria Ilza Silva e Antônio Timóteo.

\section{REFERÊNCIAS}

ANDRADE-LIMA, D. Estudos fitogeográficos de Pernambuco. Arquivo do Instituto de Pesquisa Agronômica. Secretaria da Agricultura, Indústria e Comércio, Pernambuco, Brasil, 5, 305-341, 1960.

ARZABE, C. Reproductive activity patterns of anurans in two different altitudinal sites within the Brazilian Caatinga. Revista Brasileira Zoologia, 16(3), 851-864, 1999.

ARZABE, C.; SKUK, G.; SANTANA, G. G.; DELFIM, F. R.; LIMA, Y. C. C.; ABRANTES, S. H. F. Herpetofauna da área do Curimataú, Paraíba. Análise das variações da biodiversidade do Bioma Caatinga: suporte a estratégias regionais de conservação. Brasília: Ministério do Meio Ambiente, p. 264-280, 2005.

BORGES-NOJOSA, D.; SANTOS, E. M. Herpetofauna da área de Betânia e Floresta, Pernambuco. In: ARAÚJO, F. S.; RODAL, M. J.; BARBOSA, M. R. V. (Org.). Análise das variações da biodiversidade do bioma Caatinga: suporte a estratégias regionais de conservação. Brasília: MMA, 2005. p. 276-289.

DAJOZ, R. Ecologia geral: Petrópolis: Vozes, 472 p. 1983.

FREITAS, M. A.; SILVA, T. F. S. Guia ilustrado: a herpetofauna das caatingas e áreas de altitudes do Nordeste brasileiro: 384 p. USEB, Pelotas, 2007.

IBGE, 2015. Fundação Instituto Brasileiro de Geografia e Estatística. Disponível em: http://ibge.gov.br/cidadesat/xtras/perfil.php?lang=_EN\&codmun=261247\&search=pernambuco|san ta-cruz-da-baixa-verde. Acesso em: 31 maio 2016.

ICMBIO - Instituto Chico Mendes de Conservação da Biodiversidade. 2014. Lista de Espécies Ameaças. Disponível em: http://www.icmbio.gov.br/portal/. Acesso em: 20 maio 2016.

IUCN - União Internacional para a Conservação da Natureza. 2015. IUCN Red List of Threatened Species. Version 2011.2. Disponível em: http://www.iucnredlist.org. Acesso em: 20 maio 2016.

LOEBMANN, D.; HADDAD, C. F. B. Amphibians and reptiles from a highly diverse area of the Caatinga domain: composition and conservation implications. Biota Neotrópica, 10(3), 227-256, 2010.

MOURA, G. J. B. Herpetologia no estado de Pernambuco: 1-442. IBAMA, Brasília, 2011.

NOGUEIRA, C.; VALDUJO, P. H.; FRANÇA, F. G. R. Habitat variation and lizard diversity in a Cerrado area of Central Brazil. Studies on Neotropical Fauna and Environment, 40(2), 105-112, 2005.

PÔRTO, K. C.; CABRAL, J. J. P.; TABARELLI, M.; (Org.). Brejos de altitude em Pernambuco e Paraíba: história natural, ecologia e conservação: 1-324. Ministério do Meio Ambiente, Brasília, 2004.

RODRIGUES, M. T. 2003a. A fauna de répteis e anfíbios das caatingas. In: Workshop Biodeversidade da Caatinga. Petrolina, PE. 2003. Disponível em: http://www.biodiversitas.org/caatinga/relatorios/repteis_anfibios.pdf. Acessado em: 14 maio 2016. 
RODRIGUES, M. T. Anfíbios e répteis: áreas prioritárias para a conservação da Caatinga. In: BRASIL PROBIO - Projeto de Conservação e Utilização Sustentável da Diversidade Biológica Brasileira. MMA. Avaliação e ações prioritárias para a conservação da biodiversidade do Bioma Caatinga, 2003b.

SANTOS, E. M.; CARNAVAL, A. C. O. Q. Anfíbios anuros do Estado de Pernambuco. In: TABARELLI, M; SILVA, J.M.C. (orgs.). Diagnóstico da biodiversidade de Pernambuco, Editora Massagana, Recife, 2001. p. 529-533.

SEMAS - Secretaria de Meio Ambiente e Sustentabilidade de Pernambuco. Espécies Ameaçadas. 2015. Disponível em: http://www.semas.pe.gov.br/web/semas. Acesso em: 20 maio 2016.

VIEIRA, W. L.; ARZABE, C.; SANTANA, G. G. Composição e distribuição espaço-temporal de anuros no Cariri Paraibano, Nordeste do Brasil. Oecologia Australis, 11(3), 383-396, 2007. 